2

9 Running head: Name Conditioning and ERPs

10

Word count: Main text 3377 words; Abstract 195 words; total 4590 words

\author{
Boris Kotchoubey (1) and Yuri G. Pavlov $(1,2)$
}

(2) Department of Psychology, Ural Federal University, Jekaterinburg, Russia boris.kotchoubey@uni-tuebingen.de

\section{Conflict of interests: None}

\title{
Name Conditioning in Event-Related Brain Potentials
}

(1) Institute of Medical psychology and Behavioral Neurobiology, University of Tübingen, Germany

Corresponding author: B. Kotchoubey, Institute of Medical psychology and Behavioral Neurobiology, University of Tübingen, Silcherstr. 5, 72076 Tübingen, Germany, phone +4970712974221, e-mail 


\section{Abstract}

22 Four experiments are reported in which two harmonic tones (CS+ and CS-) were paired with a

23 participant's own name (SON) and different names (DN), respectively. A third tone was not paired with

24 any other stimulus and served as a standard (frequent stimulus) in a three-stimuli oddball paradigm. The

25 larger posterior positivity (P3) to SON than DN, found in previous studies, was replicated in all

26 experiments. Conditioning of the P3 response was albeit observed in two similar experiments (1 and 3),

27 but the obtained effects were weak and not identical in the two experiments. Only Experiment 4, where

28 the number of CS/UCS pairings and the Stimulus-Onset Asynchrony between CS and UCS were increased,

29 showed clear CS+/CS- differences both in time and time-frequency domains. Surprisingly, differential

30 responses to CS+ and CS- were also obtained in Experiment 2, although SON and DN in that experiment

31 were masked and never consciously recognized as meaning words (recognition rate 0/63 participants).

32 The results are discussed in the context of other ERP conditioning experiments and, particularly, the

33 studies of non-conscious effect on ERP. Several further experiments are suggested to replicate and

34 extend the present findings and to remove the remaining methodological limitations.

37 Key words: Classical conditioning, Consciousness, Masking, Own name, P3, Passive oddball 
Effects of classical conditioning on human Event-Related Brain Potentials (ERPs) have been examined in a number of studies (for review, see Christoffersen \& Schachtman, 2016; Miskovic \& Keil, 2012). Most of them used highly aversive unconditioned stimuli (UCS) (e.g., Hermann, Ziegler, Birbaumer, \& Flor, 2000; Pizzagalli, Greishar, \& Davidson, 2003). As a rule, CS are complex visual stimuli, e.g., faces (Begleiter, \& Platz, 1969) or words (Montoya, et al., 1996). Very few studies employed both CS and UCS of auditory modality (Hugdahl \& Norby, 1991; Heim \& Keil, 2006; Pauli \& Röder, 2008; Juan et al., 2016).

Because auditory classical conditioning, due to its technical simplicity, can be applied in children and severely disabled individuals, and because using highly aversive UCS in these groups is ethically problematic, looking for other kinds of UCS is important. Having in mind the potential application in patients with severe brain damage, in the present study we intended to explore the effect of classical conditioning on ERP to simple stimuli. Relatively simple harmonic tones were chosen as CS, based on the finding that harmonic tones elicit more distinct and stable ERP effects than sine tones in both healthy

51 individuals (Tervaniemi et al., 2000) and neurological patients (Kotchoubey et al., 2003). An individual's

52 own name, which has been suggested to possess particular significance for the individual, was used as a non-aversive UCS. The effects of a subject's own name (SON) on ERPs have been established in normal populations (Fischler et al., 1987) and severely brain-injured patients (Perrin et al., 2006), in waking state

55 (Holeckova et al., 2006) and during sleep (Perrin, Garcia-Larrea, Mauguiere, \& Bastuji, 1999). In a threestimulus oddball, in which SON and a control stimulus (usually, a different name: DN) are presented as

57 two rare stimuli, SON elicits a larger P3 component than DN (e.g., Perrin et al., 2006; Kotchoubey et al., 58 2004). We expected to obtain a similar effect in response to harmonic tones (presented as CS) paired 59 with names.

\section{Methods: General}


61 Three different groups of healthy participants took part in the study: one group (nine males and 14

62 females, aged 22-29) in Experiment 1, the second group (nine males and 13 females, aged 22-29) in

63 Experiments 2 and 3, and the third group (twelve males and 13 females, aged 19-42) in Experiment 4. In

64 the second group Experiment 2 always preceded Experiment 3. Data of two males in Experiment 2 were

65 excluded (thus the group contained 7 males).

None of the participants had had any disease of the nervous system or hearing disorders in the past, or

reported use of any drugs during the last week before the experiment. Participants were seated in a

comfortable chair and asked to close their eyes and to listen attentively to the stimuli. Informed consent of Tübingen.

The EEG in all experiments was recorded using 64 active ActiCHamp electrodes (Easycap GmbH, electrooculagram were also recorded. The resistance was below $15 \mathrm{kOhm}$. Online reference was at $\mathrm{Cz}$, offline re-referenced to average mastoids. The digitalization rate was $1000 \mathrm{~Hz}$.

Off-line inspection of the recordings revealed in some traces poor data quality in one or two of the 64 channels. These channels were replaced with interpolation of the adjacent electrodes. After this, an Independent Component Analysis (ICA) was employed for each participant to separate and remove activity due to ocular artifacts using the AMICA algorithm (Palmer et al., 2012). Components clearly related to eye movements were removed using EEGLAB. Additionally, components that were mapped onto one electrode and could be clearly distinguished from EEG signals were subtracted from the data.

81 EEG segments that still contained artifacts after ICA correction were dismissed. The ERPs were filtered 
separately for the first, second, and third thirds of the whole sequence of 400 stimuli. The three periods, corresponding to stimuli $1-133$, stimuli $134-266$, and stimuli $267-400$, will be referred to as T1, T2, and T3, respectively. Each average included at least 18 (usually 20) CS+ and CS-.

The amplitudes of ERP components were measured as the area under the curve within the time windows slightly different for UCS (N1: 70-120 ms, P3a: 240-300 ms, P3b: 300-400 ms, Late Time Window [LTW]: 400-600 ms) and CS (N1: 100-140 ms, P3a: 220-280 ms; P3b: 280-380 ms; LTW: 380-550 ms). The LTW was not designated as " $\mathrm{P}$ " or " $\mathrm{N}$ " because the amplitude was negative in anterior but positive in posterior leads. The time-frequency analysis was performed using Morlet wavelet by means of the Fieldtrip toolbox and followed the method of Cavanagh et al. (2010). The entire epochs were defined as [-1500 2500] ms to avoid edge artifacts. Baseline correction and decibel normalization was performed in respect to [-400 -100] ms interval. After a visual exploration of grand averages across all subjects, ms, 9-12 Hz) and "LTW" (400-650 ms, 6-8 Hz).

For brevity, the present report describes only those data that are related to the critical comparison between the CS+ and CS- responses at the midline electrodes $\mathrm{Fz}, \mathrm{Cz}$, and $\mathrm{Pz}$, were the effects were best pronounced. The statistical analysis was performed using a repeated measures ANOVA with factors Stimulus, Site, and Time. When appropriate, we used Greenhouse-Geisser non-sphericity correction for 101 degrees of freedom.

\section{Experiment 1}

\section{Methods}

ERPs were recorded to three chords, each consisting of five harmonic tonal frequencies (e.g., 330, 660, 
times with three different names (DN). All names were spoken with the official German pronunciation by a female speaker, not familiar to any participant. The control names originated from the same pool of the most frequent German names used for each subject's own name. They always had a very similar duration as the own name (means $669 \mathrm{~ms}$ and $676 \mathrm{~ms}$ ) and contained the same number of syllables. The standard was presented 21 times, not accompanied by any other stimulus. Tone duration was $200 \mathrm{ms,}$

112 and the intensity was $75 \mathrm{~dB}$ above the average threshold. The stimulus-onset-asynchrony (SOA) within a

113 pair tone-name was $300 \mathrm{~ms}$. The SOA after a tone-word pair was $1700-1800 \mathrm{~ms}$, and after standards it

114 was 1150-1250 ms. All stimuli were presented binaurally through aerodynamic earphones, in a

115 pseudorandomized order, in which none of the three tones appeared more than three times in a row.

116 In the test phase, which followed immediately after the acquisition phase, the standard was presented

117280 times, and CS+ and CS- 60 times each. No other stimuli were presented. The SOA varied between

118950 and $1050 \mathrm{~ms}$. The order of presentation was randomized except that CS+ and CS- could not be

119 delivered more than twice in a row.

\section{Results}

121 All participants reported after the experiment, that they had heard "two or three" different harmonic

122 tones, and that at the beginning one of the tones was linked to their own name, and another tone, to

123 other names.

124 The acquisition phase replicated the already known effect of a larger P3 to SON. The effect was most

125 clear in the P3a window $\left(F(1,22)=11.55, p=.003, \eta^{2}=.34\right)$. Also in the LTW, the amplitude was negative 126 to $\mathrm{DN}$ but positive to SON at $\mathrm{Cz}$ and $\mathrm{Pz}$, yielding a significant Stimulus $\mathrm{x}$ Site interaction: $\mathrm{F}(2,44)=5.37, \mathrm{p}$

$127=.014, \eta^{2}=.20$. Importantly, the P3a amplitude was larger to CS+ than CS- (mean amplitudes 3.47 versus 

waveforms are shown in Figures 1 and 2.

\section{Methods}

133 In Experiment 2 the names were completely masked while preserving their acoustical features. The first

$13425 \%$ of time points of an original name were multiplied by a linearly spaced vector of coefficients from

1351.5 to 0 , and the remaining $75 \%$ points were set to 0 . Then, the first $25 \%$ of time points of the same

136 name played backwards were multiplied by a linearly spaced vector of coefficients from 0 to 1.5 , and the

137 last $75 \%$ time points remained unchanged. Finally, the two files were added. This technique permitted to

138 attain the same intensity-by-time dynamics as in the original names. In a pilot experiment the stimuli

139 were presented to forty healthy participants. None of them was able to recognize any name including

140 their own.

\section{Results}

142 Participants reported that they had heard "two or three" different harmonic tones, and that at the

143 beginning of the stimulation also other stimuli had been presented that sounded like non-

144 comprehendible words of an exotic (non-European) language.

145 Despite the lack of subjectively perceived differences between the two UCS, P3b had a larger amplitude

146 to SON than DN, particularly at Pz (main Stimulus effect: $F(1,20)=5.04, p=.035, \eta^{2}=.20$; Stimulus $\times$ Site

147 interaction: $\left.F(2,40)=5.85, p=.017, \eta^{2}=.23\right)$. P3a, in contrast to Experiment 1 , did not differ between

148 SON and DN. During the test phase, N1 and P3a were significantly larger to CS+ than CS- $(F(1,20)=20.21$,

$\mathrm{p}<.001, \eta^{2}=.50 ;$ and $4.95, \mathrm{p}=.038, \eta^{2}=.20$, for $\mathrm{N} 1$ and $\mathrm{P} 3 \mathrm{a}$, resp.). 


\section{Methods}

152 Although CS+ and CS- differed in the perceived pitch, in Experiment 3 they also were delivered

153 monaurally in two different ears to further increase their discriminability. The side of presentation was

154 counterbalanced among the participants.

\section{Results}

156 Participants reported after the experiment that they had heard three different harmonic tones. They

157 were aware of the association between tones and names.

158 Both P3a and P3b were larger to SON than DN at Pz (Stimulus $x$ Site interaction: $F(2,42)=4.54, p=.03, \eta^{2}$

$159=.18$; and 4.75, $\mathrm{p}=.026, \eta^{2}=.18$; for P3a and P3b, resp.). The main effect of Stimulus at Pz was

160 significant $(p<.02)$ for both components. The general main Stimulus effect approached significance for

161 P3b: $\left.F(1,21)=3.87, p=.058, \eta^{2}=.15\right)$. In the test phase, the amplitude in the LTW was more positive to

$162 \mathrm{CS}+$ than $\mathrm{CS}-\left(\mathrm{F}(1,21)=4.68, \mathrm{p}=.042, \eta^{2}=.18\right) . \mathrm{P} 3 \mathrm{a}$, in contrast to Experiments 1 and 2 , appeared to be

163 slightly larger to CS- than CS+, but this difference did not attain significance.

\section{Experiment 4}

\section{Methods}

166 This Experiment entailed only one phase, during which the standard was presented 280 times, and CS+

167 and CS- 60 times each. Tone duration was $100 \mathrm{~ms}$ including $5 \mathrm{~ms}$ rise/fall phase. All CS were followed by

168 the corresponding UCS with SOA of $600 \mathrm{~ms}$. This design aimed at the recording of the late ERP

169 components to CS immediately during acquisition, because in the other experiments (with SOA of 300

$170 \mathrm{~ms}$ ) they could be measured only in the test phase.

\section{Results}


172 SON elicited a larger P3b than $\operatorname{DN}\left(F(1,24)=5.08, p=.034, \eta^{2}=.18\right)$, and this difference steadily

173 decreased with time (Stimulus $x$ Time interaction: $F(2,48)=3.40, p=.040, \eta^{2}=.13$ ). The LTW was

174 consistently negative to DN, but negative at $\mathrm{Fz}$ and positive in T1 at Pz (thus zero on average) to SON,

175 resulting in a main effect of Stimulus $\left(F(1,24)=8.68, p=.007, \eta^{2}=.27\right)$ and a Stimulus $\times$ Site $x$ Time

176 interaction $\left(F(4,96)=3.51, p=.023, \eta^{2}=.13\right)$.

177 CS+ elicited more positive P3b $\left(F(1,24)=4.40, p=.047, \eta^{2}=.16\right)$ and more positive LTW amplitude

$178\left(F(1,24)=7.83, p=.01, \eta^{2}=.25\right)$ than CS-. When only the first $20 \mathrm{CS}+/$ SON and CS-/DN pairs are taken

179 into the analysis (for comparison with the other experiments in which CS+/SON and CS-/pairs were

180 presented 20 times each), the same difference is also significant for $\mathrm{P} 3 \mathrm{a}: \mathrm{F}(1,24)=5.36, \mathrm{p}=.03, \eta^{2}=.18$.

181 Time-frequency analysis

182 As can be seen in Figure 3, the upper theta activity in the LTW in response to the tones was suppressed

183 as compared with the baseline. In experiments 2 and 4, this suppression was stronger to CS- than CS + at

$184 P z$ (main effect of Stimulus in Experiment 2: $F(1,20)=6.12, p=.02, \eta^{2}=.23$; Stimulus $x$ Site interaction in

185 Experiment 4: $F(2,42)=3.52, p=.05, \eta^{2}=.13$; main effect of Stimulus at Pz: $F(1,24)=4.69, p=.03, \eta^{2}=$

186 .16). Two further effects were observed in Experiment 4. First, alpha activity in the P3 window was

187 desynchronized after CS+ but not after CS- (main effect of Stimulus: $F(1,24)=4.67, p=.04, \eta^{2}=.16$ ).

188 Second, theta activity was strongly suppressed in the LTW at Pz after DN, but less after SON (Stimulus x

189 Site interaction: $\left.F(2,42)=3.76, p=.04, \eta^{2}=.14\right)$.

190 Discussion

191 The results are an intriguing mix of expected and surprising findings. First, we replicated the already

192 known fact of an increased P3 to SON as compared with DN in a different paradigm. The increase was

193 observed in all three experiments in which participants were able to recognize their own names. We 
194 hypothesized that the effect would be partially transferred to the related CS. In fact, CS+ elicited a larger

195 positivity in the P3a time window than CS- during acquisition in Experiments 1 and 4, but not in

196 Experiment 3 where it was expected as well. We also expected that the stable reinforcement in

197 Experiment 4 would result in an increase of the difference between CS+ and CS-, and this hypothesis was

198 confirmed. Due to the short CS/UCS SOA we could not record ERP components later than P3a in the

199 acquisition phase of Experiments 1-3. In Experiment 4, with a longer SOA, we found that the short-living

200 effect in the P3a time window stabilized and extended to the later time intervals between 300 and 600

$201 \mathrm{~ms}$

To summarize, these findings indicate that classical conditioning of electrophysiological responses using the own name as UCS is possible. The differential responses to CS+ and CS- in Experiment 4 were demonstrated in both time (i.e., late ERP positivities) and time-frequency domains (i.e., higher theta but lower alpha activity to CS+ than CS-). Importantly, the better differentiation between the two CS was found in the same experiment in which the two UCS were also best distinguished. Generally, however, learning effects were relatively weak and unstable. Experiment 4, in which the clearest learning effects were obtained, did not contain extinction. The only effect that survived extinction was the differential response in the LTW in Experiment 3. Therefore, we do not believe that the paradigm can be used in

210 clinical applications in its present form; rather, further methodological work is needed. Another

211 limitation concerns the theoretical interpretation. Although the effect was primarily concentrated

212 around P3a, we cannot rule out that other ERP components (e.g., P2 or P3b) also contributed to its

213 generation. Also this issue should be followed in further experiments.

214 On the background of these largely predicted findings, the results of Experiment 2 were fully

215 unexpected. Remember the masked names from this experiment were presented to a total of 63

216 individuals (40 participants of the pilot experiment +22 participants of Experiment $2+$ the first author

217 who was unaware about masking developed by the second author), and none of them (0/63) recognized 
any name including their own. The stimuli were not even perceived as meaningful words. ERP, however,

219 demonstrated a significantly larger positivity to SON than DN. Notably, this differential response was

220 about 100 ms later than in all three experiments where SON was consciously recognized, indicating the

221 presence of some additional processing operation(s). Although conditional responses in Experiment 2 did

222 not differ during acquisition, the test phase revealed a significant P3a effect, quite similar to that in the

223 acquisition phase of Experiment 1 and 4 , and additionally, a very strong $\left(\eta^{2}=.50\right)$ enhancement of the

224 N1 amplitude to CS+ as compared with CS-. This N1 effect was very consistent at the individual level,

225 being observed in 20 of the 22 participants.

226 During acquisition, SON was presented as UCS+ with the same frequency as three DN as UCS-. Therefore,

227 each individual DN was presented three times less frequently than SON. Although this fact is a

228 methodological limitation of the present study, it can hardly be responsible for any expected or

229 surprising result. Firstly, this arrangement was the same in all four experiments, but the results were

230 different. Secondly, N1 and P3a are expected to be larger to rare than frequent stimuli. If N1 is

231 superposed by a Mismatch Negativity, this wave is also larger to rare than frequent stimuli. On this basis

232 we might expect larger amplitudes to CS- (previously linked to rare DN) than to CS+ (previously linked to

233 the more frequent SON), but the opposite was found. Only the non-significant increase of P3a in

234 Experiment 3 would be in line with the frequentist interpretation. But even in this case such

235 interpretation meets a considerable problem: it is fully unclear why ERP responses differ between the

236 two CS having equal frequencies (and only linked to stimuli of different frequencies), whereas these

237 responses do not differ between the two UCS having different frequencies.

238 If explanations related to experimental methodology are rejected, the only possible interpretation of the

239 data of Experiment 2 remains that participants subconsciously distinguished between SON and DN even

240 though they did not recognize them. This is particularly possible because our masking technique assured

241 the similar intensity/time function of unmasked and masked UCS. If, for example, a sound file consisted 
of 100 data points, the first point was the same in masked and unmasked stimuli, the second point

243 contained $95.8 \%$ common information, the third point $91.7 \%$, etc. It may, therefore, be speculated that

244 participants unconsciously recognized different personal significance of the stimuli even though they did

245 not identify their content.

246 The fact that stimuli that are not consciously recognized can nonetheless elicit significant ERP effects has

247 been shown in numerous studies (reviews Shevrin, 2001; Dehaene et al., 2006). A discussion of whether

248 all kinds of learning in the brain can happen outside awareness (e.g., Hassin, 2013) will go far beyond the

249 topic of classical conditioning, where subliminal effects on ERPs have repeatedly been shown, most

250 recently by Beckes, Coan and Morris (2013). Most such subliminal effects are similar to (but usually

251 weaker than) the corresponding effect of supraliminal, consciously perceived stimuli, while in the current

252 study the results of Experiment 2 differ qualitatively from those of other experiments. A direct

253 comparison between different studies is hardly possible due to the huge variability of techniques making

254 stimuli non-recognizable. However, some studies demonstrated non-conscious ERP effects different

255 from, stronger or even faster than similar effect to consciously perceived stimuli (e.g., Dehaene et al.,

256 1998; Eimer \& Schlaghecken, 1998; Williams et al., 2004). For instance, Eimer and Schlaghecken (1998)

257 showed nearly equally strong, but opposite in direction, effects of masked and unmasked stimuli on the

258 Lateralized Readiness Potential, an ERP index of motor preparation. All these and similar findings pose

259 serious theoretical problems for both strong and weak single-process models of classical conditioning

260 discussed by Lovibond and Shanks (2002) and favor their third, dual-process model.

261 Taking into account that this is the first indication of conditioning effects of a subject's own name,

262 suggestions for further experiments can be made. First, an extensive acquisition phase like in our

263 Experiment 4 should be combined with a test phase like in Experiments 1 and 3. Second, an additional

264 experiment using one DN should clarify the issue of stimulus frequencies. Third, regarding the putative

265 non-conscious conditioning, different kinds of masking should be compared in respect of their ERP 
effects, and sophisticated techniques of debriefing should be used to explore the possible role of poorly

267 formulated affective features of stimuli.

Acknowledgement: The study was supported by the German Research Society (Deutsche

270 Forschungsgemeinschaft), Grant KO-1753/13. We thank Ms. Janna Holst for her help in running the 271 experiments.

\section{References}

Begleiter, H., \& Platz, A., (1969). Evoked potentials: modifications by classical conditioning. Science, 166, 503-516.

Cavanagh, J. F., Frank, M. J., Klein, T. J., \& Allen, J. J. B. (2010). Frontal theta links prediction errors to behavioral adaptation in reinforcement learning. Neurolmage, 49(4), 3198-3209. associative learning in humans. Behav. Brain Res., 296, 211-232. Moortele, P-F ., \& Le Bihan, D. (1998). Imaging unconscious semantic priming. Nature, 395, 597subliminal processing: A testable taxonomy. Trends Cogn. Sci., 10(5), 204-211. 
Fischler, I., Jin, Y.S., Boaz, T.L., Perry, N.W., \& Childers, D.G. (1987). Brain potentials related to seeing one's own name. Brain Lang., 30, 245-262.

Hassin, R. R. (2013). Yes it can: On the functional abilities of the human unconscious. Perspect. Psychol. Sci., 8, 195-207.

Heim, S., \& Keil, A. (2006). Effects of classical conditioning on identification and cortical processing of speech syllables. Exper. Brain Res., 175, 411-424.

Hermann, C., Ziegler, S., Birbaumer, N., \& Flor, H. (2000). Pavlovian aversive and appetitive odor conditioning in humans: subjective, peripheral and electrocortical changes. Exper. Brain Res, 132, 203-215.

Holeckova, I., Fischer, C., Giard, M.H., Delpuech, C., \& Morlet, D. (2006). Brain responses to a subject's own name uttered by a familiar voice. Brain Res., 1082, 142-152.

Hugdahl, K., \& Norby, H. (1991). Hemisphere differences in conditional learning: An ERP study. Cortex, $27,557-570$.

Juan, E., Nguissi, N. A. N., Tzovara, A., Viveic, D., Rusca, M., Oddo, M., Rossetti, A., \& De Lucia, M. (2016). Evidence of trace conditioning in comatose patients revealed by the reactivation of EEG responses to alerting sounds. Neuroimage, 141, 530-541.

Kotchoubey, B., Lang, S., Herb, E., Maurer, P., \& Birbaumer, N. (2004). Reliability of brain responses to the own name in healthy subjects and patients with brain damage. In N.C. Moore \& M.K. Arikan (Eds.), Brainwaves and Mind: Recent Advances (pp. 75-80). New York: Kjellberg, Inc. 
Lovibond, P. F., \& Shanks, D. R. (2002). The role of awareness in Pavlovian conditioning: Empirical evidence and theoretical implications. J. Exp. Psychol. Animal Behav. Proc., 28, 3-26.

Miskovic, V., \& Keil, A. (2012). Acquired fears reflected in cortical sensory processing: A review of electrophysiological studies of human classical conditioning. Psychophysiology, 49, 1230-1241.

Montoya, P., Larbig, W., Pulvermüller, F., Flor, H., \& Birbaumer, N. (1996). Cortical correlates of semantic classical conditioning. Psychophysiol., 33, 644-649.

Palmer, J. A., Kreutz-Delgado, K., \& Makeig, S. (2012). AMICA: An adaptive mixture of independent component analyzers with shared components. Swartz Center for Computational Neuroscience, University of California San Diego, Tech. Rep. https://sccn.ucsd.edu/ jason/amica_a.pdf.

Pauli, W., \& Röder, B. (2008). Emotional salience changes the focus of spatial attention. Brain Res., 1214, 94-104.

Perrin, F., Garcia-Larrea, L., Mauguiere, F., \& Bastuji, H. (1999). A differential brain response to the subject's own name persists during sleep. Clin. Neurophysiol., 110, 2153-2164.

Perrin, F., Schnakers, C., Schnabus, M., Degueldre, C., Goldman, S., Bredart, S., et al. (2006). Brain response to one's own name in vegetative state, minimally conscious state, and locked-in syndrome. Arch. Neurol., 63, 562-569. aversive classical conditioning: high density event-related potential and brain electrical 
bioRxiv preprint doi: https://doi.org/10.1101/194134; this version posted September 26, 2017. The copyright holder for this preprint (which was not certified by peer review) is the author/funder, who has granted bioRxiv a license to display the preprint in perpetuity. It is made available under aCC-BY 4.0 International license.

330 Tervaniemi, M., Ilvonen, T., Sinkkonen, J., Kujala, A., Alho, K., Huotilainen, M., et al. (2000). Harmonic partials facilitate pitch discrimination in humans: Electrophysiological and behavioral evidence. Neurosci. Lett., 279, 29-32.

333 Williams, L. M., Liddell, B. J., Rathjen, J., Brown, K. J., Gray, J., Phillips, M., Young, A., \& Gordon, E. (2004). Mapping the time course of nonconscious and conscious perception of fear: An integration of central and peripheral measures. Hum. Brain Map., 21, 64-74. 


\section{Figure Legends}

337 Figure 1. Grand average ERP obtained in the acquisition phase of the four experiments in response to 338 CS+/SON (solid line) and CS-/DN (dashed line) combinations. Solid vertical lines indicate the onset of CS,

339 and dotted vertical lines, the onset of UCS. Note that the rightmost column shows ERP averaged across

340 the first one-third of Experiment 4, to make the number of trials comparable for all experiments.

341 Positivity is plotted upwards.

342 Figure 2. Experiment 1 to 3: Grand average ERP waveforms obtained in the test phase. Experiment 4:

343 Grand average across the whole experiment. The number of included trials is similar for all four

344 experiments. Labelings are similar to Figure 1.

345 Figure 3. Results of the time-frequency analysis. Blue colors indicate power decrease, and yellow/red

346 colors indicate power increase, as compared with the baseline. The color scale is presented in $\mathrm{dB}$ (that is,

347 a value of $+10 \mathrm{~dB}$ would mean the increase of the EEG power in ten times). Note that the epochs are

348 broader than those shown in Figures 1 and 2, because the time-frequency analysis has, by definition, a

349 lower temporal resolution than the analysis in time domain. No significant effects were found in

$350 \quad$ Experiments 1 and 3. 


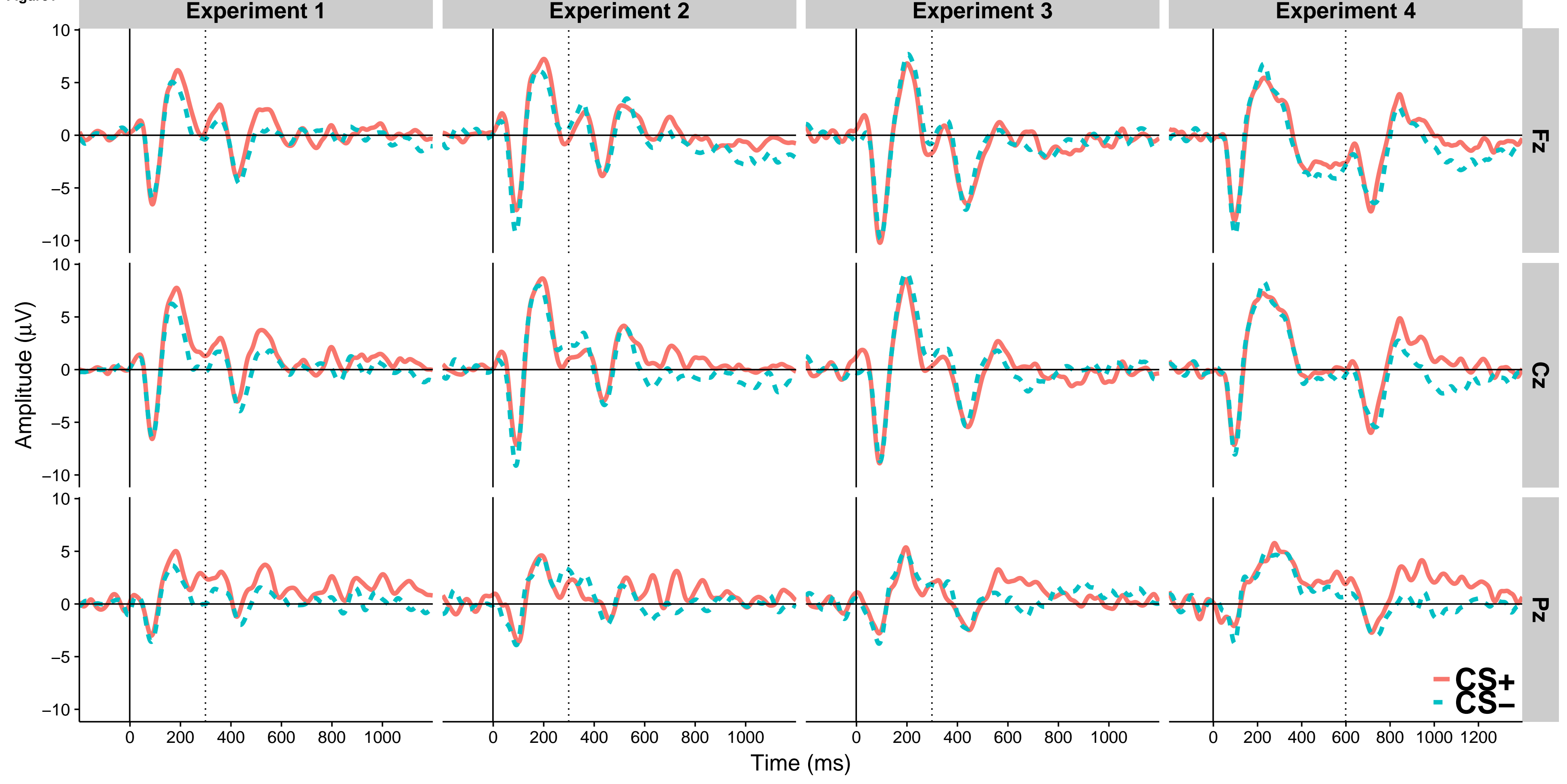



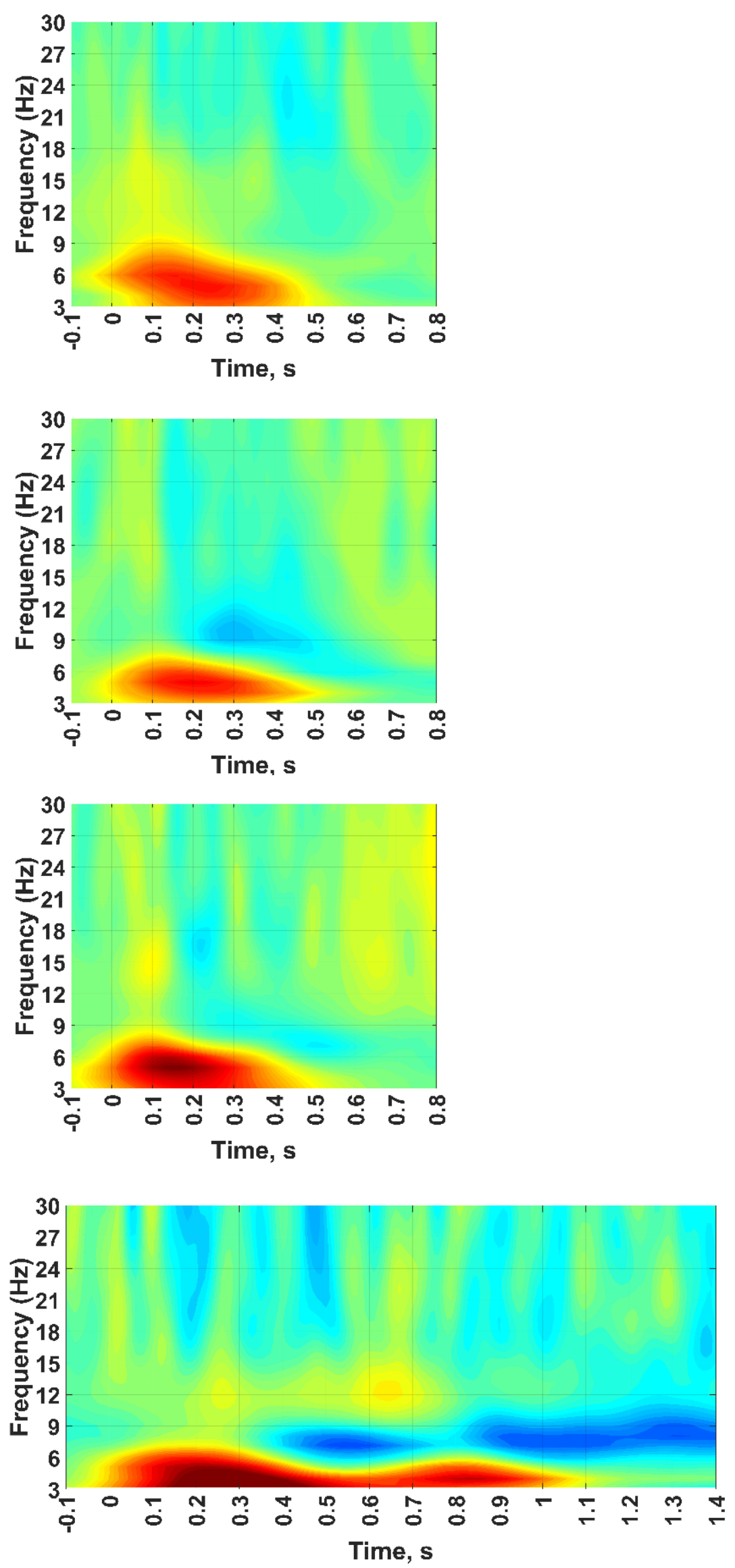
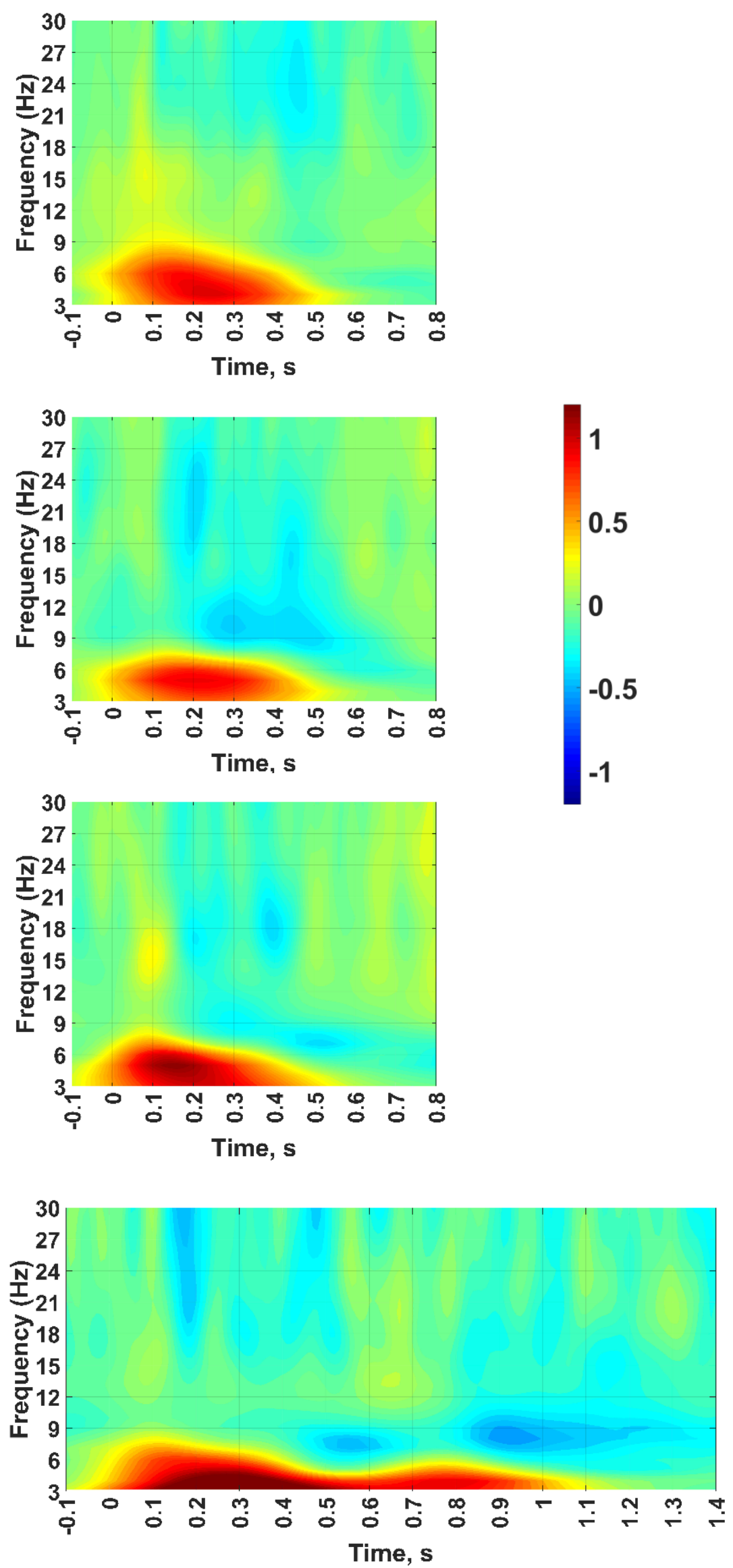